\title{
Deep Brain Stimulation Abolishes Slowing of Reactions to Unlikely Stimuli
}

\author{
Chrystalina A. Antoniades, ${ }^{1 \star}$ Rafal Bogacz, ${ }^{1,3 \star}$ Christopher Kennard, ${ }^{1}$ James J. FitzGerald, $, 1,2$ Tipu Aziz, ${ }^{1,2}$ \\ and Alexander L. Green ${ }^{1,2}$ \\ ${ }^{1}$ Nuffield Department of Clinical Neurosciences and 2Nuffield Department of Surgical Sciences, University of Oxford, Oxford OX3 9DU, United Kingdom, \\ and ${ }^{3}$ Medical Research Council Anatomical Neuropharmacology Unit, Department of Pharmacology, University of Oxford, Oxford OX1 3TH, United \\ Kingdom
}

The cortico-basal-ganglia circuit plays a critical role in decision making on the basis of probabilistic information. Computational models have suggested how this circuit could compute the probabilities of actions being appropriate according to Bayes' theorem. These models predict that the subthalamic nucleus (STN) provides feedback that normalizes the neural representation of probabilities, such that if the probability of one action increases, the probabilities of all other available actions decrease. Here we report the results of an experiment testing a prediction of this theory that disrupting information processing in the STN with deep brain stimulation should abolish the normalization of the neural representation of probabilities. In our experiment, we asked patients with Parkinson's disease to saccade to a target that could appear in one of two locations, and the probability of the target appearing in each location was periodically changed. When the stimulator was switched off, the target probability affected the reaction times (RT) of patients in a similar way to healthy participants. Specifically, the RTs were shorter for more probable targets and, importantly, they were longer for the unlikely targets. When the stimulator was switched on, the patients were still faster for more probable targets, but critically they did not increase RTs as the target was becoming less likely. This pattern of results is consistent with the prediction of the model that the patients on DBS no longer normalized their neural representation of prior probabilities. We discuss alternative explanations for the data in the context of other published results.

Key words: deep brain stimulation; eye movements; Parkinson's; prior probabilities; reaction times

\section{Introduction}

The basal ganglia are a group of subcortical nuclei that play a critical role in action selection (Redgrave et al., 1999), and are affected by Parkinson's disease (PD; Obeso et al., 2000). An important role is played by the subthalamic nucleus (STN), which sends widespread projections to neurons in other nuclei of the basal ganglia (Parent and Hazrati, 1995) including the output nuclei (the internal segment of the globus pallidus and the substantia nigra pars reticulata). Although STN neurons are glutamatergic, their activity effectively inhibits movements, because they excite the inhibitory output nuclei. It has been suggested that the function of the STN is to inhibit the neural representations of actions other than the one being selected (Mink, 1996) and to slow down the initiation of movement in the presence of conflict-

\footnotetext{
Received March 17, 2014; revised June 9, 2014; accepted June 24, 2014.

Author contributions: C.A.A. designed research; C.A.A. and A.L.G. performed research; C.A.A., R.B., J.J.F., and A.L.G. analyzed data; C.A.A., R.B., C.K., J.J.F., T.A., and A.L.G. wrote the paper.

C.A.A. was supported by a grant from the Wellcome Trust (073735), by the National Institute of Health Research, by the Oxford Biomedical Research Centre, and by the Dementias and Neurodegenerative Diseases Research Network. R.B. was supported by EPSRC Grant EP/1032622/1 and the Medical Research Council.

${ }^{*}$ C.A.A. and R.B. contributed equally to this work.

The authors declare no competing financial interests.

Correspondence should be addressed to Chrystalina Antoniades, Nuffield Department of Clinical Neurosciences, University of 0xford, Oxford 0X3 9DU, UK. E-mail: Chrystalina.antoniades@clneuro.ox.ac.uk.

DOI:10.1523/JNEUROSCI.1065-14.2014

Copyright $\odot 2014$ the authors $\quad 0270-6474 / 14 / 3410844-09 \$ 15.00 / 0$
}

ing sensory information (Frank, 2006, 2007b; Zaghloul et al., 2012).

It has also been suggested that many aspects of the anatomy and physiology of the basal ganglia are exactly those required to compute the probabilities of actions being appropriate in a given context according to Bayes' theorem (Bogacz and Gurney, 2007; Ditterich, 2010; Bogacz and Larsen, 2011; Lepora and Gurney, 2012). One model (Bogacz and Larsen, 2011) describes how the equation of Bayes' theorem (Fig. 1A), can be mapped on the anatomy of the circuit (Fig. $1 B$; see Materials and Methods). In the model the feedback from the STN ensures that the represented probabilities of actions add up to 1 . Because of this normalization, the model can compute the actual probabilities of actions being appropriate, rather than just which action is most likely. This allows judgment of whether the probability of the "winning" action is sufficiently high to select it, or whether more sensory input should be gathered. It has been shown that selecting actions when their normalized probabilities reach an appropriately chosen threshold allows maximization of reward rate for correct choices in a wide range of tasks (Bogacz, 2009). The model predicts that disrupting information processing in the STN should disrupt the normalization of the neural representation of probabilities.

To test the above prediction we used the fact that in tasks involving saccades to a target (Fig. 2), the probability of the target 
A

\section{Bayes' theorem}

$P\left(A_{i} \mid S\right)=\frac{P\left(A_{i}\right) P\left(S \mid A_{i}\right)}{P(S)}$

Normalization

$P(S)=\sum_{i=1}^{N} P\left(A_{i}\right) P\left(S \mid A_{i}\right)$

\section{Log of Bayes' equation}

$$
\begin{aligned}
\log P\left(A_{i} \mid S\right)= & \log P\left(A_{i}\right)+\log P\left(S \mid A_{i}\right) \\
& -\log P(S)
\end{aligned}
$$

B
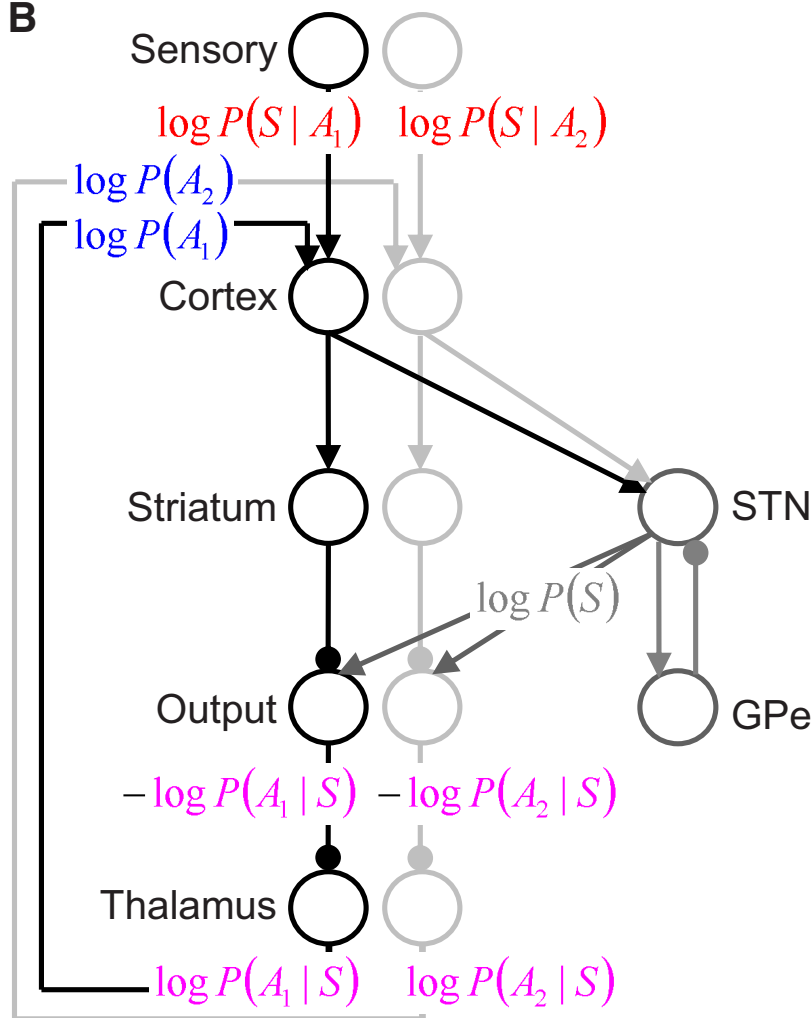

Figure 1. Mapping of Bayes' theorem on the cortico — basal— ganglia-thalamic circuit. $\boldsymbol{A}$, Bayes' theorem in its original (top) and logarithmic (bottom) form. $\boldsymbol{B}$, Mapping of Log of Bayes' equation on the subset of cortico — basal- ganglia-thalamic circuit. Black and light gray circles denote neuronal populations selective for two actions situated in different brain regions labeled next to the circles. Output, output nuclei of the basal ganglia; cortex, - frontoparietal cortical areas including neurons selective for actions. Arrows denote excitatory connections, while lines ended with circles denote inhibitory connections.

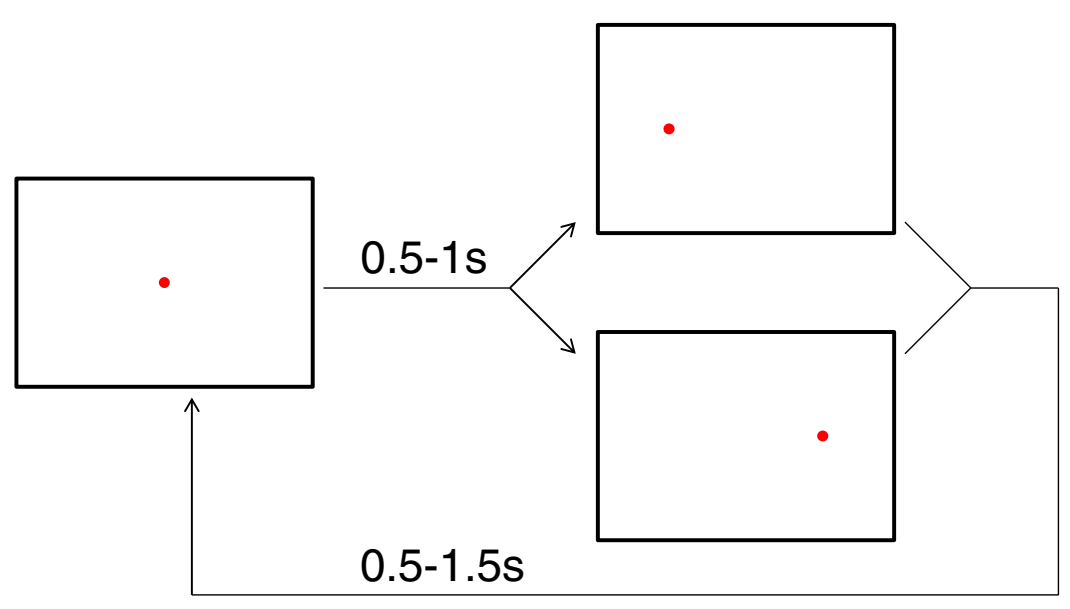

Figure 2. Time course of a trial. A trial started with presentation of a central fixation point. After a random interval between 0.5 and $1 \mathrm{~s}$, the fixation point disappeared and a target appeared on the left or right to which participants were required to saccade. The probability of the target appearing on a particular side was constant within a block, but differed between the blocks. After the saccade, the target remained for a random period between 0.5 and $1.5 \mathrm{~s}$, and then disappeared while the central fixation appeared. Once the participants saccaded back to the central fixation a new trial began.

appearing in a particular location estimated by a participant is reflected in the saccadic reaction time (RT) to this target (Carpenter and Williams, 1995; Forstmann et al., 2010; Mulder et al., 2012). In particular, if participants learn that one of two possible target locations is more likely, the RT to the likely target decreases, and importantly the RT to the less likely target increases. This suggests that as the estimated probability of one location increases, the estimated probability of the other location de- creases, which is consistent with the two probabilities being normalized. The model (Bogacz and Larsen, 2011) predicts that when information processing in the STN is disrupted, the normalization of probabilities will be disrupted and the change in RT for one target may no longer be accompanied by the opposite change in RT for the other. In line with these predictions, our results suggest that in patients with STN deep brain stimulator (DBS) systems for Parkinson's disease the normalization of probabilities is intact when the stimulator is switched off but fails when it is turned on.

\section{Materials and Methods}

Participants. The present study was approved by the Regional Ethics Committee (REC reference 04/Q0406/60) and was conducted in the John Radcliffe Hospital, Oxford, UK. Six PD patients (Table 1) and six male healthy agedmatched controls (age: 46, 58, 67, 68, 70, and 70) participated in the study. All participants were recruited from the Oxford Functional Neurosurgery unit and gave their informed consent after the procedures had been explained to them. All PD patients underwent bilateral DBS of the STN and the system implanted was connected to a stimulator set to deliver $60 \mu$ s or $90 \mu$ s pulses at $130 \mathrm{~Hz}$ (Table 2). No additional inclusion or exclusion criteria were applied in selecting patients for this study. 
Table 1. Details of patients tested

\begin{tabular}{|c|c|c|c|c|c|c|}
\hline $\begin{array}{l}\text { Patient } \\
\text { number }\end{array}$ & Age & Sex & $\begin{array}{l}\text { Year of } \\
\text { diagnosis }\end{array}$ & Medications & $\begin{array}{l}\text { UPDRS Part } \\
\text { III-DBS ON }\end{array}$ & $\begin{array}{l}\text { UPDRS Part } \\
\text { III-DBS OFF }\end{array}$ \\
\hline 1 & 56 & M & 1996 & Stalevo, amantadine & 22 & 34 \\
\hline 2 & 47 & M & 2005 & Sinemet Plus, entacapone & 13 & 76 \\
\hline 3 & 65 & M & 2000 & Stalevo, ropinorole, pergoline, rasagiline & 6 & 33 \\
\hline 4 & 68 & M & 2006 & Sinemet Plus, amantadine & 19 & 41 \\
\hline 5 & 65 & M & 2002 & Rasagiline, Sinemet Plus, apomorphine & 10 & 43 \\
\hline 6 & 70 & M & 2000 & Madopar, entacapone & 3 & 16 \\
\hline
\end{tabular}

Unified Parkinson's Disease Rating Scale (UPDRS) scores are with DBS ON and OFF (patients were on their medication in both cases). Sinemet Plus contains a combination of levodopa and carbidopa; Stalevo contains levodopa, carbidopa, and entacapone; and Madopar contains levodopa and benserazide.

Table 2. Stimulation parameters

\begin{tabular}{|c|c|c|c|c|c|c|c|}
\hline \multirow{2}{*}{$\begin{array}{l}\text { Patient } \\
\text { number }\end{array}$} & \multirow[b]{2}{*}{ Side } & \multicolumn{3}{|c|}{$\begin{array}{l}\text { Lead tip coordinates (mm with } \\
\text { respect to mid AC-PC line) }\end{array}$} & \multirow[b]{2}{*}{ Contacts used } & \multirow[b]{2}{*}{ Volts } & \multirow{2}{*}{$\begin{array}{l}\text { Pulse width } \\
\text { (all at } 130 \mathrm{~Hz} \text { ) }\end{array}$} \\
\hline & & AP & Lateral & Vertical & & & \\
\hline 1 & L & -6.6 & -11.2 & -6.2 & $1-$ & 2.6 & 60 \\
\hline \multirow[t]{2}{*}{2} & L & -5.3 & -10.5 & -4.1 & $0-$ & 3.2 & 90 \\
\hline & $\mathrm{R}$ & -6.6 & 11.4 & -6.7 & $10-$ & 3.3 & 90 \\
\hline 3 & L & -4.9 & -9.5 & -8.5 & $0-$ & 2.5 & 60 \\
\hline 4 & $\mathrm{R}$ & -3.7 & 12.5 & -3.4 & $10-$ & 2.9 & 60 \\
\hline \multirow[t]{2}{*}{5} & L & -6.5 & -13.4 & -4.4 & $0-, 1+$ & 3.7 & 60 \\
\hline & $\mathrm{R}$ & -6.2 & 13.5 & -3.6 & $8-, 9-, 10+$ & 3.4 & 60 \\
\hline \multirow[t]{2}{*}{6} & L & -4.7 & -10.5 & -6.4 & $1-, 2+$ & 2.0 & 60 \\
\hline & $\mathrm{R}$ & -3.1 & 11.7 & -6.2 & $9-, 10+$ & 2.7 & 90 \\
\hline
\end{tabular}

$A C-P C$, anterior-posterior commissure; $L$, left; $R$, right.

Task. Visually guided horizontal saccades were recorded using a miniaturized infrared $1 \mathrm{kHz}$ saccadometer, low-pass filtered at $250 \mathrm{~Hz}$ with 12-bit resolution (Antoniades et al., 2012). Patients wore the oculometer on their head, secured by an elastic strap and resting on the bridge of the nose; three built-in, low-power lasers projected red $13 \mathrm{~cd} \mathrm{~m}^{-2}$ spots subtending $\sim 0.1$ degrees in a horizontal line in the midline at \pm 10 degrees (Ober et al., 2003). Because the stimuli move exactly with the head, no head restraint was necessary. Each trial started with a central fixation light, and then after a random delay a second target light appeared randomly either to the left or right. The participants were instructed to make a saccade as quickly and as accurately as they could to the second target light (Fig. 2). Trials were conducted in five blocks and the probabilities of the target appearing on the left or right differed between the blocks of trials. The different blocks had the following fractions of trials with the target appearing on the left: $10,25,50,75$, and $90 \%$. The number of trials for each block varied according to the probability being tested ( 250 trials for the 10 and $90 \%$ blocks, 200 trials for the 25 and $75 \%$ blocks, and 160 trials for the $50 \%$ blocks). In half of the PD cases and healthy controls the order of blocks was $10,25,50,75$, and $90 \%$, and in the other half it was 90 , $75,50,25$, and $10 \%$.

All testing was performed in the on-medication state. DBS patients were tested first with the system switched on. The system was then switched off and after $30 \mathrm{~min}$ the patients were retested with the system remaining off for the duration of testing.

Statistical analysis. To assess statistically if the RTs of control participants depended on the target probability, the median RTs were subjected to an ANOVA, with one factor of probability. To analyze statistically RTs of patients, their median RTs were subjected to a two-factor ANOVA, with factors of probability and DBS setting. The key aspect of this analysis is the test for the interaction between effects of probability and DBS setting, because such interaction indicates that the RTs depend on probability in a different way when DBS is on and when it is off, as predicted by the model.

Review of the computational model. The model we used in simulations of our task is an extension of a previously published model (Bogacz and Larsen, 2011). This model assumes that during action selection, the cortico-basal-ganglia circuit computes for each available action, $A_{i}$, the probability that this action is appropriate in the current context. These probabilities are constantly updated on the basis of sensory information, $S$, until for any action its probability exceeds a threshold of confidence. The way that the probabilities of actions should be updated on the basis of sensory input is described by Bayes' theorem shown in the top equation in Figure $1 A$. It simply says that to compute the updated or posterior probability of action $P\left(A_{i} \mid S\right)$, one needs to multiply the previous or prior probability $P\left(A_{i}\right)$ by the probability of the sensory input $S$ appearing on trials on which action $A_{i}$ is rewarded, denoted $P\left(S \mid A_{i}\right)$, which could have been learned from experience. Additionally, to ensure that the posterior probabilities add up to 1 , this product is divided by a normalization term $P(S)$ equal to the sum of corresponding products across all $N$ actions (Fig. $1 A$, middle equation). Bayes theorem includes multiplication and division, which are not natural operations for neurons, but this problem can be solved by taking the logarithm of both sides of the equation, giving the bottom equation in Figure $1 \mathrm{~A}$. Thus, if the neurons have firing rates proportional to the logarithms of probabilities then the update according to Bayes' theorem can be performed just using addition and subtraction.

Figure $1 B$ illustrates how the logarithmic form of Bayes' equation is mapped in the model on the subset of the cortico-basal - ganglia-thalamic circuit. According to the mapping, frontoparietal cortical neurons add the logarithms of prior probability provided by a feedback from the thalamus to the logarithms of the likelihood of sensory information, which under certain assumptions can be provided in the firing rate of sensory cortical neurons (Jazayeri and Movshon, 2006; Zhang and Bogacz, 2010). The STN and external segment of the globus pallidus (GPe) compute the logarithm of the normalization term (by summation and nonlinear transformations of their input from cortex). The output nuclei receive excitation from the STN and inhibition from the cortex via the striatum so, according to the bottom equation in Figure $1 A$, their activity in the model is proportional to the negative of the logarithms of posterior probability. The thalamus receives inhibition from the output nuclei so its activity is proportional to the logarithms of the posterior probability, and these values are sent to the cortical neurons, as they become the basis of the computation for the next time step.

The equations describing the model (labeled by the part of the circuit they describe) are as follows: 


$$
\begin{gathered}
\text { Cortical integrators : } I N T_{i}(0)=\log 1 / N+c \\
I N T_{i}(t)=T H_{i}(t-1)+g x_{i}(t) \text {, for } t>0 \\
\text { Input from STN and GPe : } S G(t)=\log \sum_{i=1}^{N} \exp I N T_{i}(t) \\
\text { Output nuclei : } O T_{i}(t)=-\operatorname{INT}_{i}(t)+S G(t) \\
\text { Thalamus: } \operatorname{TH}_{i}(t)=c-\operatorname{OUT}_{i}(t) .
\end{gathered}
$$

In the above equations, $N$ denotes the number of available alternatives, and since the task involved a choice between the two targets, $N=2$. The activity of cortical integrators is initialized to a value proportional to the logarithm of prior probability of actions $P\left(A_{i}\right)$. At the start of a simulated trial, the activity of cortical integrator neurons is initialized just on the basis of the available number of alternatives (Eq. 1) - the process of setting the initial values of the integrators before the target onset will be described later. Thus assuming that there are $N$ available alternatives and all of them are equally likely, $P\left(A_{i}\right)=1 / N$. Note that since $1 / N$ is lower than $1, \log 1 / N$ is negative. Thus a positive constant $c$ is added in Equation 1 to ensure that cortical firing rates are non-negative (but as we show below its value does not affect the activity in the output nuclei and hence decision times; in the simulations we set $c=3$ ). In the subsequent time steps $t$ the activity of integrators selective for alternative $i$ reflects the feedback from the thalamus increased by sensory input $x_{i}(t)$, weighted by a gain parameter $g$ (Eq. 2). In our simulations of the DBS switched-off condition, the gain parameter was set to $g=1$. Equation 3 states the function of cortical input computed by the STN and GPe, and it has been shown that the neurons in these areas have precisely the connectivity and the nonlinear response properties required to perform this computation (Bogacz and Gurney, 2007; more detailed explanation in Bogacz, 2009). The output nuclei compute the differences between the inputs from the STN and cortical integrators (via the striatum). Finally, the output nuclei inhibit the thalamus. The choice is made in the model when the activity of one of the populations in the output nuclei decreases below Threshold, which is consistent with experimental data suggesting that movements are initiated when the inhibitory neurons in the output nuclei have their activity reduced, thereby disinhibiting their targets (Deniau and Chevalier, 1985). Since the output nuclei have activity proportional to the negative logarithm of the probability of action (Fig. $1 B$ ), the choice is made in the model when the probability for one of the actions increases above the threshold.

In the absence of input the activity in the model does not change, while when sensory input is present, the model updates represented probabilities of actions as described in Figure 1, and at each time step the output nuclei have activity proportional to the negative of the current estimates of probabilities of actions being appropriate (see Fig. $1 B$ ). According to Equations 1 and 3 , the feedback provided by the STN at time $t=0$ is equal to the following:

$$
S G(0)=\log \sum_{i=1}^{N} \exp (c+\log 1 / N)=\log \left(\exp c \sum_{i=1}^{N} 1 / N\right)=c .
$$

The activity of the thalamus in the model is (according Eqs. 4-6):

$$
\begin{array}{r}
T_{i}(0)=c-\left(-I N T_{i}(0)+S G(0)\right)=c+\log 1 / N+c-c \\
=\log P\left(A_{i}\right)+c .
\end{array}
$$

Note that the feedback provided by the thalamus is exactly the same as the initial activity of cortical integrators; thus in the absence of sensory input the activities do not change in this model. If in a next time step when the sensory input is $g x_{i}(t)=\log P\left(S \mid A_{i}\right)+k$ (where $k$ is a constant added to ensure the input is non-negative), the feedback provided by the STN becomes:

$$
S G(t)=\log \sum_{i=1}^{N} \exp \left(c+\log P\left(A_{i}\right)+\log P\left(S \mid A_{i}\right)+k\right)=\log P(S)+c
$$

The activity of the output nuclei becomes (according to Eq. 4 and Bayes' theorem):

$$
\begin{aligned}
\operatorname{OUT}_{i}(t)=-\left(c+\log P\left(A_{i}\right)+\log P\left(S \mid A_{i}\right)+\right. & k)+\log P(S)+c+k \\
= & -\log P\left(A_{i} \mid S\right) .
\end{aligned}
$$

The logarithm of the posterior probability computed above is then fed back to the cortical integrators, as it becomes a priori for the next time step.

Although the framework of accumulation of sensory information is most naturally applicable to tasks in which the information forming the basis for decisions is spread in time (Gold and Shadlen, 2007; Yang and Shadlen, 2007), work in mathematical neurophysiology has established that even in simple reaction tasks, like the one in our study, the distributions and patterns of RTs are best explained by models assuming accumulation of sensory information (Carpenter and Williams, 1995). Consequently, below we present how the model described above can be used to simulate choices in our task.

Setting the initial activities of integrators. When simulating a trial in a block with target 1 being more likely with probability $P_{1}$, we provide input at the first time step $x_{1}(1)$ such that the neurons in the output nuclei selective for target 1 represent the correct probability, i.e., such that the activity of the output nuclei is $\operatorname{OUT}_{1}(1)=-\log P_{1}$. Substituting Equations 1 and 3 into 4 we see that the activity of the output nuclei at the first time step is as follows:

$$
\begin{array}{r}
\operatorname{OUT}_{1}(1)=-\left(\log \frac{1}{2}+x_{1}(1)\right)+\log \left(\exp \left(\log \frac{1}{2}+x_{1}(1)\right)\right. \\
\left.+\exp \left(\log \frac{1}{2}\right)\right) .
\end{array}
$$

Substituting $\operatorname{OUT}_{1}(1)=-\log P_{1}$ in the above equation and solving for $x_{1}(1)$, we obtain the value of input required to set the initial activity of the integrators:

$$
x_{1}(1)=\log \frac{P_{1}}{1-P_{1}} .
$$

Thus in the first step of the simulation the input given by Equation 11 is provided. While simulating trials in blocks with target 2 being more likely, an analogous input is also provided.

Simulating a trial. The simulation of a trial was divided into time steps with length $d t=5 \mathrm{~ms}$. After simulated stimulus onset one of the sensory inputs was set to $x_{i}(t)=A d t$, where $A$ is a parameter describing the amount of sensory input. For simplicity no input was provided by the sensory neurons selective for the other target, and no noise was added. The RT was taken as the number of time steps required for one of the output populations to decrease below Threshold, multiplied by $d t$, and increased by a nondecision time $T_{0}$.

Simulating DBS. While simulating the effects of DBS in the model of Figure $1 B$, we followed an approach similar to a previous study (Coulthard et al., 2012) and set the input from the STN to a constant value, i.e., $S G(t)=c$. Recall from Equation 6 that $c$ is equal to the feedback provided by the STN in the model in the absence of sensory input, and this particular level of STN activity allows the network to maintain its activity levels in the absence of sensory input (Eq. 7). Although the firing rate of STN neurons is likely to be higher with DBS on than with DBS off, computational models suggest that the downstream structures (e.g., thalamus) may adapt to increased STN activity due to DBS to be able to transmit information on motor command (Rubin et al., 2012) and produce similar levels of activity as in a healthy state. Such adaptation is captured in our model by setting the effective input from STN to $S G(t)=c$.

Moreover, the lack of additional inhibition provided normally by the STN when the sensory input is present resulted in the model becoming highly reactive to sensory inputs. Thus we assumed that the network naturally adapts to be less reactive, and sets the gain parameter to a lower value $g_{D B S}$ (the value of this parameter was found for which the model best reproduced the experimental data; see the section below). 
A

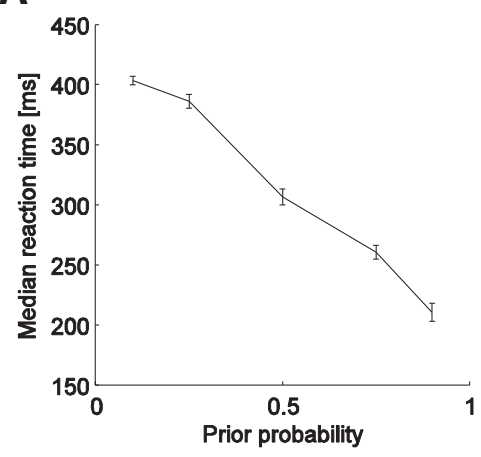

D

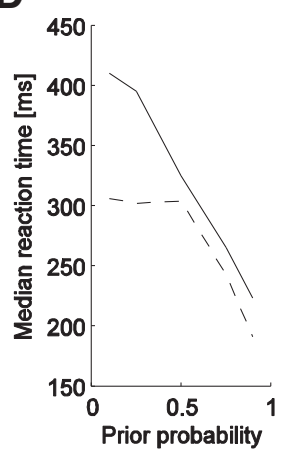

B

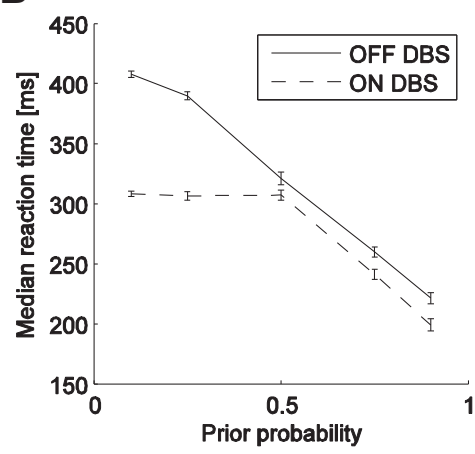

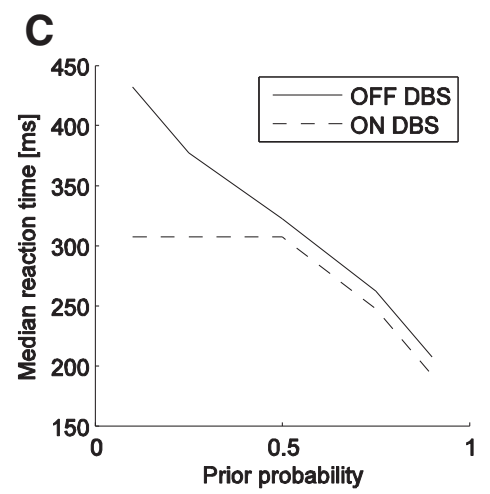

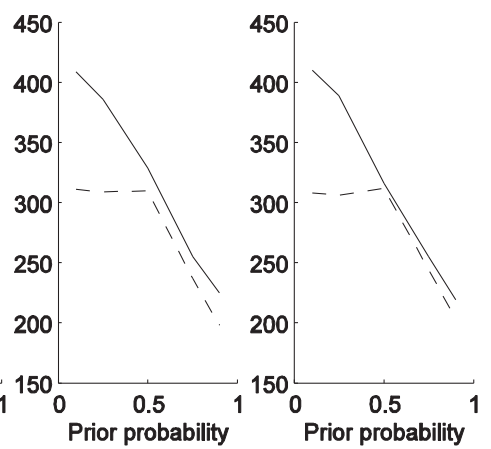

Figure 3. Average median reaction time as a function of target probability. $\boldsymbol{A}$, Control participants. $\boldsymbol{B}, \mathrm{DBS}$ patients. $\boldsymbol{C}$, Simulations of the computational model. In $\boldsymbol{A}$ and $\boldsymbol{B}$ the error bars indicate SD of the median RT across the participants. D, Data from DBS patients plotted individually.

Finding model parameters. Values for the free parameters of the model, $A$, Threshold, $T_{0}, g_{D B S}$, were found for which the model produced a similar behavior to the patients. A single set of model parameters has been used to fit the data off and on DBS, and the simulations of different DBS settings used the same values of parameters $A$, Threshold, $T_{0}$, and only differed in the value of parameter $g$, and the function computed by the STN (see previous section). We found the parameter values that minimized the squared differences between the average RTs of the patients and RTs in the model, summed over all 10 conditions (2 DBS conditions $\times 5$ Prior probability conditions). We fitted the model to the data pooled across patients, as all patients produced very similar patterns of RTs. The search was performed using the simplex algorithm (Nedler and Mead, 1965). The lowest value of the summed squared error was found for the following parameter values: $A=19.57$, Threshold $=0.0385$, $T_{0}=152 \mathrm{~ms}, g_{D B S}=0.222$, and Figure $3 C$ shows the RTs for these values. Nevertheless, during the optimization we noticed that there were many different sets of parameters giving a close match between the model and the data. This is not surprising, given that many parameters have similar effects on RTs produced by the model, in particular RTs can be increased by increasing Threshold or $T_{0}$, or by decreasing $A$ or $g$. Furthermore, the difference between the RTs to unlikely and likely responses can be increased by increasing Threshold, or decreasing $A$ or $g$. The fitting procedure was not performed to reliably estimate the parameters of the underlying choice process, but instead to show that a set of parameters exists for which the model can reproduce the data.

\section{Results}

\section{Effects of DBS on RTs}

Figure $3 A$ shows that the probability of the target appearing in a particular location affects the RT for a saccade to be made toward that target for control participants $\left(F_{(4,25)}=1120, p<0.001\right)$. Compared with the RT in the unbiased situation ( $50 \%$ of targets to each side), the RT for targets with higher probabilities was shorter and the RT for targets with lower probabilities was longer. Similar dependence of RT on probability was present for PD patients off stimulation (Fig. $3 B$ ). In contrast, for patients on stimulation, the RT was decreased when the probability increased $>50 \%$, but the RT did not increase as the probability reduced below $50 \%$, exactly as predicted above (effect of probability: $F_{(4,50)}=3001, p<0.001$; stimulation: $F_{(1,50)}=2075, p<0.001$, and their interaction: $\left.F_{(4,50)}=303, p<0.001\right)$. This pattern of RTs was consistently observed for all six patients tested.

\section{Computational model}

The lack of symmetry in the effects of high and low probability on RTs that was observed on DBS is predicted by our model assuming that disruption of information processing in the STN disrupts normalization of the neural representation of probabilities.

The computational model (Bogacz and Larsen, 2011) assumes that the prior probabilities of actions are encoded in the activities before stimulus onset of the neurons selective for the actions, but the model did not previously describe how these initial neural activities are set up. Here we extend the model to describe setting initial activities and show how it can replicate the data in Figure $3 B$. Recordings of neural activity suggest that the RT is, to a large extent, determined by the time it takes for neural activity to increase from an initial level before stimulus onset to a response threshold (Schall and Hanes, 1993). The RT depends on the probability of action, because the activity of neurons selective for a particular action before stimulus onset depends on the probability of this action being required (Basso and Wurtz, 1998, 2002; Platt and Glimcher, 1999). Thus the larger the probability of an action, the closer is the initial firing of the neurons selective for this action to the response threshold and hence the faster the RT (Fig. 4, compare $A, B$ ).

It has been suggested (Bogacz et al., 2006) that the increase in the initial firing rate of neurons representing the likely action 
A

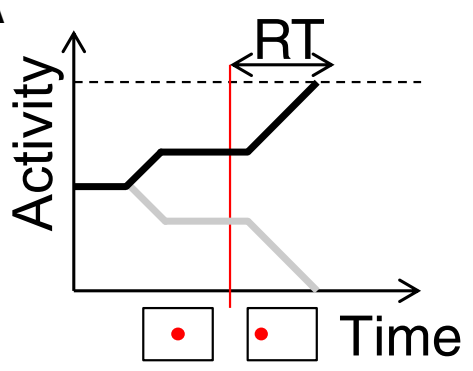

C

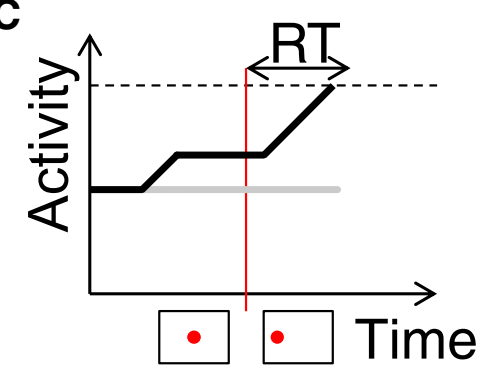

B

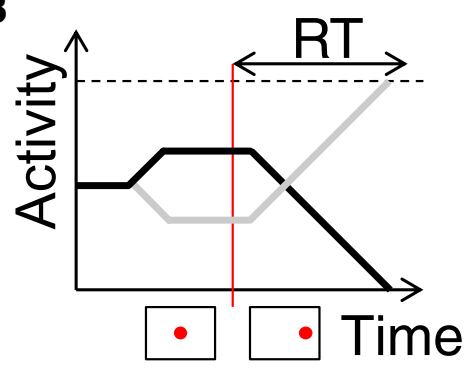

D

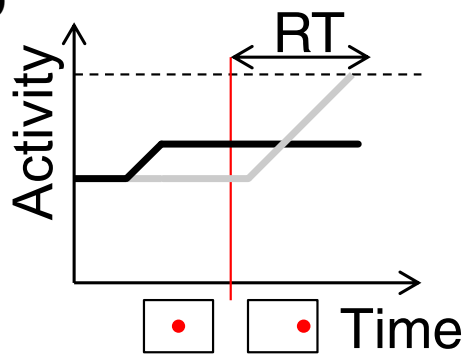

Figure 4. Schematic illustration of changes in firing rate of cortical neurons selective for eye movements to the likely (black curve) and the unlikely (gray curve) targets, as a function of time within a trial in the model. Below each graph the schematics indicate target movement to the left (likely in this example) or right (unlikely). Vertical red lines indicate target onset. Dashed black lines indicate response threshold, and arrows above them indicate the response times (which include a brief period of sensory processing, time required for activity to rise from an initial value to the threshold, and a brief period of motor command execution). $\boldsymbol{A}$ and $\boldsymbol{C}$ correspond to trials when the likely target appears, while $\boldsymbol{B}$ and $\boldsymbol{D}$ correspond to trials when the unlikely target appears. $\boldsymbol{A}$ and $\boldsymbol{B}$ correspond to DBS switched off, while $\boldsymbol{C}$ and $\boldsymbol{D}$ correspond to DBS switched on.

(Fig. 4A, B, black curves) may arise from inputs to these neurons before stimulus onset, and imaging studies have reported increased activity in several cortical areas and in the striatum after a cue indicating that one action might be more likely (Forstmann et al., 2010; Mulder et al., 2012). Consequently, in the computational model we assume that neurons selective for the likely action receive input that increases their activity. After such an increase in the activity of one neural population, the activities are normalized in the model such that the represented probabilities of all alternative actions add up to 1 . In particular, such normalization results in a decrease of the activity of neurons representing the unlikely alternative (Fig. $4 A, B$, gray curves) below the level for unbiased choices. The model predicts that this normalization is critically dependent on the STN, and without the correct STN feedback the initial activity of neurons representing the unlikely alternative does not change before stimulus onset (Figs 4C,D, gray curves). Thus during the simulations of trials with DBS switched on, the RTs to the unlikely target (Fig. 4D) are not longer than on trials where both target locations are equally likely.

A detailed description of the model is provided in Materials and Methods. The model produces a pattern of RTs in simulations (Fig. $3 C$ ) very similar to that observed in the experiment (Fig. 3B).

\section{Discussion}

The different effects of DBS on the RT to low- and highprobability targets (Fig. $3 B$ ) suggests different neural mechanisms for the speedup of responses to likely stimuli, and the slowdown of response to unlikely stimuli seen in healthy humans (Fig. 3A). In particular, the contrast between the large effect of DBS on RTs for unlikely stimuli (that flattens the left part of the dashed curve in Fig. $3 B$ ) and the small effect on RTs for likely stimuli (that just shifts the right part of the dashed curve with respect to the solid curve) indicates that the DBS interferes to a much larger extent with the mechanism responsible for the slowdown than with the mechanism responsible for the speedup. We demonstrated in simulations that the observed results can be accounted for by a model in which the slowdown of responses to unlikely stimuli depends on feedback from the STN, which before stimulus onset lowers the activity of neurons representing the unlikely response, to normalize the represented probabilities of actions.

\section{Alternative explanations for the data}

Below we discuss three alternative explanations that can be formulated for our data and how these explanations relate to other published results. We start by considering if our data can be explained by a theory postulating that the STN postpones action execution when conflicting inputs are present (Frank, 2006, 2007b). Within this framework the selective effect of DBS on RTs to unlikely targets could be explained by suggesting that the STN is predominantly involved in trials with unlikely targets, because in these trials there is a conflict between prior belief and incoming information. Such an explanation is plausible when our data are considered on their own, but the preferential involvement of the STN during the response to unlikely targets would have to increase the RTs in these trials by means of one of two possible mechanisms. First, by increasing the threshold of activity the cortical neurons need to reach for the response to be initiated (Cavanagh et al., 2011). However, neurophysiological data show no increase in the response threshold of the cortical neurons due to changes in prior probability (Hanks et al., 2011). Second, RTs may be increased by inhibiting cortical neurons (via feedback from the thalamus), and effectively reducing the rate of rise in the activity of neurons selective for the chosen response. But an analysis of RTs from a task similar to our experiment indicates that prior probability does not change the rise rate of the decision variable (Carpenter and Williams, 1995). Instead, the neurophysiological data suggest that in simple saccadic tasks the prior probability changes the initial levels of activity of neurons selective for likely and unlikely responses before stimulus onset (Platt and Glimcher, 1999). Given these results, our data suggest that the STN is involved not only during action, as proposed by other theories (Mink, 1996; Frank, 2006), but it already plays a critical role before stimulus onset, when there was no conflict in sensory evidence in our task.

The selective effect of DBS on RTs to unlikely stimuli could also be explained by assuming that the prior probabilities for likely responses are given by striatal neurons with D1 receptors, which facilitate these actions via the direct (or "go") pathway (Fig. $5 A, B$ ), while the prior probabilities of unlikely responses are given by striatal neurons with $\mathrm{D} 2$ receptors, which inhibit the actions via the indirect (or "no go") pathway (Fig. 5C). Thus just before the stimulus onset the increased activity of striatal D1 neurons selective for the likely response results in lower inhibi- 
A

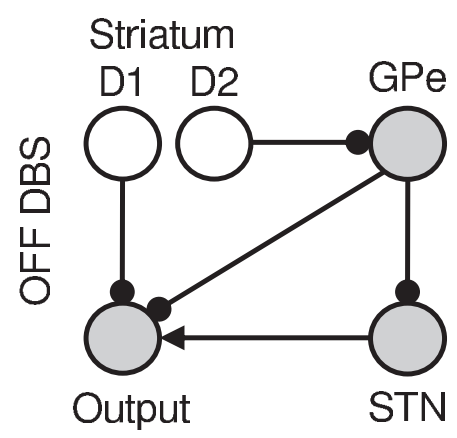

D

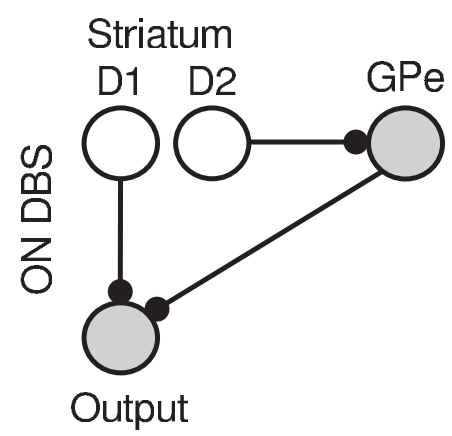

B

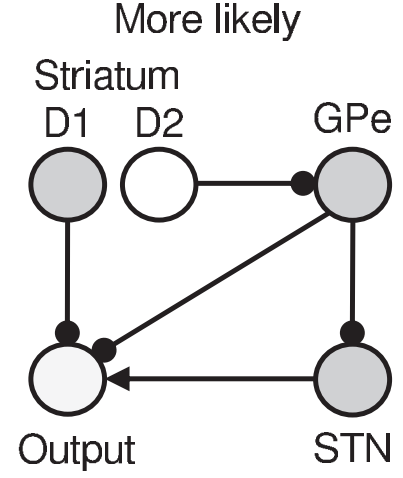

E

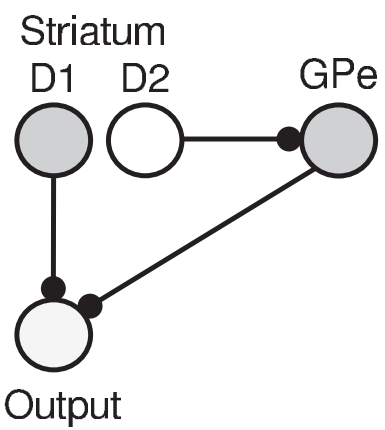

C

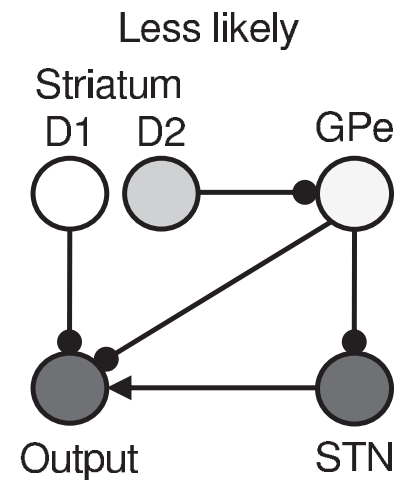

$\mathbf{F}$

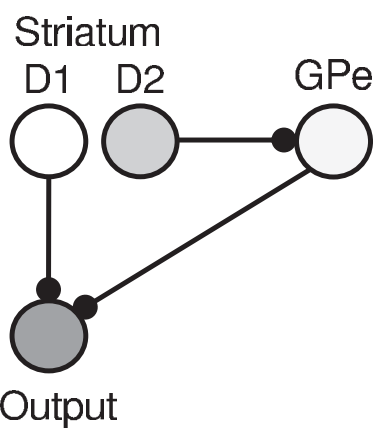

Figure 5. An alternative way of accounting for the experimental data assuming that the probabilities of actions being likely or unlikely are learned separately by striatal neurons in direct and indirect pathways, respectively. In each part, circles denote neural populations selective for one of the actions, located in different parts of the basal ganglia as indicated by the labels. The darkness of the circles indicates the activity level of the populations before stimulus onset. Arrows denote excitatory connections, while lines ended with circles denote inhibitory connections. The three columns correspond to blocks where the considered action has $50 \%$ probability of being required $(\boldsymbol{A}$ and $\boldsymbol{D})$, is more likely $(\boldsymbol{B}$ and $\boldsymbol{E})$, or is less likely $(\boldsymbol{C}$ and $\boldsymbol{F})$. The two rows correspond to DBS being turned OFF and ON. Since the STN is disrupted with DBS turned ON, the input from STN is not shown in $\boldsymbol{D}-\boldsymbol{F}$. It is also assumed that the output nuclei adapt to changed input from the STN when DBS is $0 \mathrm{~N}$, and thus the output nuclei in $\boldsymbol{A}$ and $\boldsymbol{D}$ have same level of activity.

tion from neurons in output nuclei selective for this action (Fig. $5 B$ ), and faster RTs, while the increased activity of striatal D2 neurons selective for unlikely response increases inhibition of this response by output nuclei (Fig. 5C) and RT for this response. Since the STN is part of the indirect pathway but not the direct pathway, disrupting its information processing could selectively affect the slowing down for unlikely responses (Fig. 5D-F). However, the indirect pathway also includes a route that does not involve the STN (from striatum to GPe and then to output nuclei), thus under the above hypothesis one might still expect some slowdown for the unlikely stimuli even when the STN is disrupted (Fig. $5 F$ ), while the data suggest that DBS completely abolishes slowing down for unlikely stimuli.

Alternatively, the reported data could be explained by a model in which the STN mediates the inhibition between neurons selective for the two actions as illustrated in Figure 4, but in which the STN neurons are linear and do not necessarily represent the Bayesian normalization term. However, the STN neurons have nonlinear response properties (Hallworth et al., 2003; Wilson et al., 2004) that precisely match those required to compute the normalization (Bogacz and Gurney, 2007).

\section{Relationship of the model to other theories}

In the computational model of Bogacz and Gurney (2007), the STN fulfills the functions assigned to it by previous theories. In particular, the STN is involved in the inhibition of nonselected actions (as proposed by Mink (1996)), because when the probability of one action increases the STN ensures that the represented probabilities of other actions decrease. Also, the STN is involved in postponing action execution in the face of conflicting information (as proposed by Frank (2006)), because when two actions receive equally high input after normalization their probabilities will each be $50 \%$ and neither will exceed a sufficiently high threshold of confidence, until the conflict is resolved. However, the model extends the description of STN function by postulating that it ensures that represented probabilities add up to one throughout the decision process.

In the model presented in this paper, we made a very simplified assumption that DBS results in a constant level of STN activity independent of its inputs. This is similar to the functional lesion used in previous computational models of the effects of DBS on decision making (Frank et al., 2007b; Coulthard et al., 2012; Green et al., 2013). Nevertheless, the effect of DBS is much more complex (Antoniades et al., 2012) and not fully understood. Refining the effects of DBS in the model would be a very interesting direction for future work.

In addition to its modulatory function during action selection, the STN has been proposed to be involved in other functions such as interruption of the current motor plan (Aron and Poldrack, 2006; Aron et al., 2007), switching from automatic to controlled behavior (Isoda and Hikosaka, 2008), or setting the speed-accuracy trade-off (Frank et al., 2007a). The model does not preclude the STN being involved in these other functions, and is complementary to these theories rather than competitive.

\section{Relationship to other experimental data}

The critical role of the basal ganglia in producing the dependence of RT on prior probabilities of movements is suggested by a study 
of a patient who had their STN and one of the output nuclei removed unilaterally (Obeso et al., 2009). The RTs of that patient were affected by prior probabilities only for the hand ipsilateral to the lesion but not for the contralateral hand (i.e., the hand normally controlled by the lesioned basal ganglia).

Our data show that STN DBS patients are impaired in inhibiting a response, which is unlikely to be correct. This may shed some light on why patients with STN DBS engage in gambling, despite a low probability of winning (Smeding et al., 2007).

It will be interesting to see whether the STN is involved in other forms of normalization of neural activity. For example, no form of reward was used in the study we describe here, and the effect of providing rewards for accurate saccades would be worthy of investigation. It is known that if in a task similar to ours the rewards for making the two directions of saccades differ, then the available rewards modulate the RTs and the neural activity before stimulus onset in a similar way to the probabilities (Platt and Glimcher, 1999). Thus, it would be logical to ask whether DBS could selectively disrupt the increase in RT for less well rewarded saccades. Such an effect of DBS might be also expected from the observation that injecting a D2 antagonist into the caudate, which presumably increases the activity in the STN, selectively increases RT for less well rewarded actions (Hikosaka, 2007). One could also examine whether the results presented in this paper generalize to more complex tasks. It has been shown in the antisaccade task that the error rate is higher when the stimulus is presented on the side of high saccade direction probability, and lower when it is presented on the side of low saccade direction probability (Koval et al., 2004). This pattern of results is naturally explained by the stimulus location probabilities modulating the initial activity of neurons selective for the corresponding directions. Based on our results one might predict that STN DBS would, by interfering with this modulation, increase the error rate to stimuli presented on the side of low saccade direction probability. Furthermore it would be interesting to see whether this would be accentuated by mixing prosaccades and antisaccades, a situation which has been shown to increase antisaccade error rate in PD. (Rivaud-Péchoux et al., 2007).

\section{Conclusion}

Our data suggest involvement of different neural mechanisms in speeding up responses to likely stimuli and slowing down responses to unlikely stimuli. The observed pattern of results was predicted by a model in which the STN normalizes the neural representation of prior probabilities. Although alternative explanations for the data can be considered, our results together with previously published data impose significant constraints on models of action selection in the basal ganglia. Establishing if, as the model suggests, the STN provides feedback proportional to the logarithm of the normalization term in Bayes' theorem will require direct recordings of neural activity in the STN during probabilistic choice tasks.

\section{References}

Antoniades CA, Buttery P, FitzGerald JJ, Barker RA, Carpenter RH, Watts C (2012) Deep brain stimulation: eye movements reveal anomalous effects of electrode placement and stimulation. PLoS One 7:e32830. CrossRef Medline

Aron AR, Poldrack RA (2006) Cortical and subcortical contributions to Stop signal response inhibition: role of the subthalamic nucleus. J Neurosci 26:2424-2433. CrossRef Medline

Aron AR, Behrens TE, Smith S, Frank MJ, Poldrack RA (2007) Triangulating a cognitive control network using diffusion-weighted magnetic resonance imaging (MRI) and functional MRI. J Neurosci 27:3743-3752. CrossRef Medline
Basso MA, Wurtz RH (1998) Modulation of neuronal activity in superior colliculus by changes in target probability. J Neurosci 18:7519-7534. Medline

Basso MA, Wurtz RH (2002) Neuronal activity in substantia nigra pars reticulata during target selection. J Neurosci 22:1883-1894. Medline

Bogacz R (2009) Optimal decision-making theories. In: Handbook of reward and decision making (Dreher J-C, Tremblay L, eds). Oxford: Academic.

Bogacz R, Gurney K (2007) The basal ganglia and cortex implement optimal decision making between alternative actions. Neural Comput 19:442477. CrossRef Medline

Bogacz R, Larsen T (2011) Integration of reinforcement learning and optimal decision-making theories of the basal ganglia. Neural Comput 23: 817-851. CrossRef Medline

Bogacz R, Brown E, Moehlis J, Holmes P, Cohen JD (2006) The physics of optimal decision making: a formal analysis of models of performance in two-alternative forced choice tasks. Psychol Rev 113:700-765. Medline

Carpenter RH, Williams ML (1995) Neural computation of log likelihood in control of saccadic eye movements. Nature 377:59-62. CrossRef Medline

Cavanagh JF, Wiecki TV, Cohen MX, Figueroa CM, Samanta J, Sherman SJ, Frank MJ (2011) Subthalamic nucleus stimulation reverses mediofrontal influence over decision threshold. Nat Neurosci 14:1462-1467. CrossRef Medline

Coulthard EJ, Bogacz R, Javed S, Mooney LK, Murphy G, Keeley S, Whone AL (2012) Distinct roles of dopamine and subthalamic nucleus in learning and probabilistic decision making. Brain 135:3721-3734. CrossRef Medline

Deniau JM, Chevalier G (1985) Disinhibition as a basic process in the expression of striatal functions. II. The striato-nigral influence on thalamocortical cells of the ventromedial thalamic nucleus. Brain Res 334:227-233. CrossRef Medline

Ditterich J (2010) A comparison between mechanisms of multi-alternative perceptual decision making: ability to explain human behavior, predictions for neurophysiology, and relationship with decision theory. Front Neurosci 4:184. CrossRef Medline

Forstmann BU, Brown S, Dutilh G, Neumann J, Wagenmakers EJ (2010) The neural substrate of prior information in perceptual decision making: a model-based analysis. Front Hum Neurosci 4:40. CrossRef Medline

Frank MJ (2006) Hold your horses: a dynamic computational role for the subthalamic nucleus in decision making. Neural Netw 19:1120-1136. CrossRef Medline

Frank MJ, Scheres A, Sherman SJ (2007a) Understanding decision-making deficits in neurological conditions: insights from models of natural action selection. Philos Trans R Soc Lond B Biol Sci 362:1641-1654. CrossRef Medline

Frank MJ, Samanta J, Moustafa AA, Sherman SJ (2007b) Hold your horses: impulsivity, deep brain stimulation, and medication in parkinsonism. Science 318:1309-1312. CrossRef Medline

Gold JI, Shadlen MN (2007) The neural basis of decision making. Annu Rev Neurosci 30:535-574. CrossRef Medline

Green N, Bogacz R, Huebl J, Beyer AK, Kühn AA, Heekeren HR (2013) Reduction of influence of task difficulty on perceptual decision making by STN deep brain stimulation. Curr Biol 23:1681-1684. CrossRef Medline

Hallworth NE, Wilson CJ, Bevan MD (2003) Apamin-sensitive small conductance calcium-activated potassium channels, through their selective coupling to voltage-gated calcium channels, are critical determinants of the precision, pace, and pattern of action potential generation in rat subthalamic nucleus neurons in vitro. J Neurosci 23:7525-7542. Medline

Hanks TD, Mazurek ME, Kiani R, Hopp E, Shadlen MN (2011) Elapsed decision time affects the weighting of prior probability in a perceptual decision task. J Neurosci 31:6339-6352. CrossRef Medline

Hikosaka O (2007) Basal ganglia mechanisms of reward-oriented eye movement. Ann N Y Acad Sci 1104:229-249. CrossRef Medline

Isoda M, Hikosaka O (2008) Role for subthalamic nucleus neurons in switching from automatic to controlled eye movement. J Neurosci 28: 7209-7218. CrossRef Medline

Jazayeri M, Movshon JA (2006) Optimal representation of sensory information by neural populations. Nat Neurosci 9:690-696. CrossRef Medline

Koval MJ, Ford KA, Everling S (2004) Effect of stimulus probability on antisaccade error rates. Exp Brain Res 159:268-272. CrossRef Medline

Lepora NF, Gurney KN (2012) The basal ganglia optimize decision making 
over general perceptual hypotheses. Neural Comput 24:2924-2945. CrossRef Medline

Mink JW (1996) The basal ganglia: focused selection and inhibition of competing motor programs. Prog Neurobiol 50:381-425. CrossRef Medline

Mulder MJ, Wagenmakers EJ, Ratcliff R, Boekel W, Forstmann BU (2012) Bias in the brain: a diffusion model analysis of prior probability and potential payoff. J Neurosci 32:2335-2343. CrossRef Medline

Nedler JA, Mead R (1965) A simple method for function minimization. Comp J 7:308-313. CrossRef

Ober JK, Przedpelska-Ober E, Gryncewicz W, Dylak J, Carpenter RS, Ober JJ (2003) Hand-held system for ambulatory measurement of saccadic durations of neurological patients. In: Modelling and measurement in medicine (Gadja J, ed) pp 187-198. Warsaw: Komitet Biocybernitykii Inzyneierii Biomedycznej.

Obeso JA, Rodrí guez-Oroz MC, Rodríguez M, Lanciego JL, Artieda J, Gonzalo N, Olanow CW (2000) Pathophysiology of the basal ganglia in Parkinson's disease. Trends Neurosci 23:S8-S19. CrossRef Medline

Obeso JA, Jahanshahi M, Alvarez L, Macias R, Pedroso I, Wilkinson L, Pavon N, Day B, Pinto S, Rodríguez-Oroz MC, Tejeiro J, Artieda J, Talelli P, Swayne O, Rodríguez R, Bhatia K, Rodriguez-Diaz M, Lopez G, Guridi J, Rothwell JC (2009) What can man do without basal ganglia motor output? The effect of combined unilateral subthalamotomy and pallidotomy in a patient with Parkinson's disease. Exp Neurol 220:283-292. CrossRef Medline

Parent A, Hazrati LN (1995) Functional anatomy of the basal ganglia. II. The place of subthalamic nucleus and external pallidum in basal ganglia circuitry. Brain Res Brain Res Rev 20:128-154. CrossRef Medline

Platt ML, Glimcher PW (1999) Neural correlates of decision variables in parietal cortex. Nature 400:233-238. CrossRef Medline
Redgrave P, Prescott TJ, Gurney K (1999) The basal ganglia: a vertebrate solution to the selection problem? Neuroscience 89:1009-1023. CrossRef Medline

Rivaud-Péchoux S, Vidailhet M, Brandel JP, Gaymard B (2007) Mixing proand antisaccades in patients with parkinsonian syndromes. Brain 130: 256-264. Medline

Rubin JE, McIntyre CC, Turner RS, Wichmann T (2012) Basal ganglia activity patterns in parkinsonism and computational modeling of their downstream effects. Eur J Neurosci 36:2213-2228. CrossRef Medline

Schall JD, Hanes DP (1993) Neural basis of saccade target selection in frontal eye field during visual search. Nature 366:467-469. CrossRef Medline

Smeding HM, Goudriaan AE, Foncke EM, Schuurman PR, Speelman JD, Schmand B (2007) Pathological gambling after bilateral subthalamic nucleus stimulation in Parkinson disease. J Neurol Neurosurg Psychiatry 78:517-519. Medline

Wilson CJ, Weyrick A, Terman D, Hallworth NE, Bevan MD (2004) A model of reverse spike frequency adaptation and repetitive firing of subthalamic nucleus neurons. J Neurophysiol 91:1963-1980. CrossRef Medline

Yang T, Shadlen MN (2007) Probabilistic reasoning by neurons. Nature 447:1075-1080. CrossRef Medline

Zaghloul KA, Weidemann CT, Lega BC, Jaggi JL, Baltuch GH, Kahana M) (2012) Neuronal activity in the human subthalamic nucleus encodes decision conflict during action selection. J Neurosci 32:2453-2460. CrossRef Medline

Zhang J, Bogacz R (2010) Optimal decision making on the basis of evidence represented in spike trains. Neural Comput 22:1113-1148. CrossRef Medline 